\title{
Mapeando colaborativamente las experiencias de educación para el desarrollo y ciudadanía global
}

\author{
Patricia Digón Regueiro', Cristina Pérez Crego², \\ Laura Cruz López ${ }^{3}$, Rosa Ma Méndez García ${ }^{4}$
}

Resumen: La cartografía social y la educación para el desarrollo y ciudadanía global (EpDCG) comparten entre sus fines la perspectiva democrática y participativa, el compromiso con la reflexión y el diálogo sobre la realidad social, la comprensión de las dinámicas de poder, la orientación hacia el cambio y transformación cara cotas más altas de justicia social. Es por ello que se eligió la sala cartográfica como instrumento para mapear experiencias de EpDCG en Galicia. La intención es visibilizar estas experiencias a la vez que se crean redes de colaboración. Las dos salas cartográficas puestas en marcha han permitido crear espacios de encuentro, deliberación y debate, que han tenido como resultado, tanto la propia construcción colaborativa de la sala, como la realización de un mapeo inicial de experiencias de EpDCG.

Palabras clave: Educación para desarrollo, ciudadanía global, cartografia social, sala cartográfica, mapeo, experiencias de EpDCG.

Fecha de recepción: I5 de marzo de 2020.

Fecha de admisión definitiva: I5 de junio de 2020.

\footnotetext{
' Doctora por la Universidad de A Coruña (UDC) y Profesora titular en la UDC.

${ }^{2}$ Doctora por la UDC y Técnico de apoyo a la investigación en la UDC.

${ }^{3}$ Doctora por la Universidad de Santiago de Compostela (USC) y Contratada Doctora en la UDC.

${ }^{4}$ Doctora por la USC y Contratada Doctora en la UDC.
} 


\section{Collaborative mapping of experiences in EDS and global citizenship}

Abstract: Social mapping and education for development and global citizenship (EDGC) share among their goals a democratic and participatory perspective, a commitment to reflection and dialogue on social reality, an understanding of power dynamics, and an orientation towards change and transformation at the highest levels of social justice. That is why the mapping room was chosen as a tool to map experiences in EDGC in Galicia. The intention is to make these experiences visible while creating collaborative networks. The two mapping rooms which have been set up have enabled the creation of spaces for meetings, discussions and debates, which have resulted in both the collaborative construction of the room itself and the initial mapping of experiences in the context of EDGC.

Key words: Education for development, global citizenship, social mapping, mapping room, mapping, experiences.

\section{Cartographie collaborative des expériences en EDD et la citoyenneté mondiale}

Résumé: La Cartographie Sociale et l'Éducation au Développement et à la Citoyenneté Mondiale partagent parmi leurs objectifs une perspective démocratique et participative, un engagement à la réflexion et au dialogue sur la réalité sociale, une compréhension de la dynamique du pouvoir, et une orientation vers le changement et la transformation aux plus hauts niveaux de la justice sociale. C'est pourquoi la salle de cartographie a été choisie comme instrument pour cartographier les expériences en EDD etECG en Galice. L'intention est de rendre ces expériences visibles tout en créant des réseaux de collaboration. Les deux salles de cartographie qui ont été mises en place ont permis de créer des espaces de rencontre, de discussion et de débat, ce qui a abouti à la fois à la construction collaborative de la salle elle-même et à la cartographie initiale des expériences dans le contexte de l'EDD et l'ECG.

Mots clé: Éducation au développement, citoyenneté mondiale, cartographie sociale, salle de cartographie, cartographie, expériences.

\section{Introducción}

Desde el año 2013 hasta la actualidad, la ONGD Solidaridad Internacional de Galicia (SIG) e investigadoras de la Facultad de Ciencias da Educación de la Universidad de A Coruña (Galicia, España) colaboran en proyectos de investigación en educación para el desarrollo y ciudadanía global (EpDCG) financiados por la Dirección General de Cooperación Exterior de la Xunta de Galicia con la Unión Europea.

Actualmente estamos llevando a cabo un nuevo proyecto titulado Investigando en el territorio para conocer y reconocer los centros educativos que trabajan en educación para el desarrollo y ciudadanía global en Galicia. Fase ly II (PR804A2018/19). 
Este proyecto es consecuencia de las anteriores investigaciones, en las que hemos constatado la importancia de superar un enfoque reduccionista de la EpDCG; la necesidad de incidir en la formación; y la existencia de un importante número de profesionales en ejercicio que están llevando a cabo experiencias relevantes tanto a nivel de aula como de centro. A lo largo de estos años, hemos entendido urgente conocer y reconocer lo que se está haciendo en la práctica, mapeando el estado de la cuestión en Galicia como marco para poder crear redes de colaboración que ayuden a impulsar la EpDCG en los centros educativos gallegos. Para llevar a cabo este mapeo, se decidió utilizar la cartografía social, y en concreto la sala cartográfica, como metodología de trabajo, al entender que la reflexión crítica, la colaboración, la participación activa y el empoderamiento definen tanto la cartografía social como la EpDCG. En este artículo nos queremos centrar en la primera fase del proyecto mostrando los resultados del primer mapeo, que entendemos como ejemplo de la importancia de la colaboración entre actores.

\section{Mapeo colaborativo para la EpDCG}

\section{I. Las relaciones entre la cartografía social y la EpDCG}

La elección de la cartografía social como método de investigación parte de nuestra clara intención de ir más allá de un diagnóstico para acercarnos a un proceso de diálogo y construcción conjunta entre actores que nos ayudará a crear puntos de encuentro y de futura colaboración. Entendemos que la cartografía social es el método más coherente con este fin y con nuestra forma de entender la EpDCG; además de responder a nuestra trayectoria de investigación, siempre orientada al estudio cualitativo.

Con la cartografía social se busca representar los fenómenos sociales en el espacio físico y temporal donde se producen, mostrándolos en un mapa que nos puede permitir comprenderlos. Lo más interesante de este método de investigación es que esta representación es creada por los protagonistas de estos fenómenos, que son los que deciden qué y cómo representarlos. La creación conjunta del mapa se convierte en un proceso de participación y diálogo que ayuda a que nos narremos y compartamos la visión de la realidad que queremos representar.

Mónica Leivas (2019) afirma que la cartografía social es una estrategia de investigación a través de la cual los participantes reflexionan sobre sus prácticas a partir 
de la construcción de un mapa. El proceso de reflexión conjunta implica desvelar nuestro punto de vista sobre lo que sucede y, como explica Rachel Fendler (2013), ayuda a analizar las cualidades subjetivas que dan significado a los lugares. $Y$ es que el acto de mapear incita a los participantes a contarse, a comunicar y compartir sus vivencias y sus prácticas, su percepción y comprensión de los espacios que habitan. En este sentido, tal como afirma Vanessa de Oliveira Andreotti (2014), el proceso de creación de mapas ayuda a indagar en narrativas individuales 0 colectivas que ponen en marcha la autoconciencia, la auto-reflexión y la auto-reflexividad. La construcción de estos mapas nos permite esclarecer nuestras propias concepciones y muestra que todas las interpretaciones se construyen social, cultural e históricamente.

De ahí el gran potencial de la cartografía social, por su capacidad de hacer visible lo invisible (Hanney, 2018). Este potencial queda articulado en tres aspectos definitorios: en el proceso de creación de los mapas, ya que lo que se busca es facilitar espacios de encuentro y mediación de significados (Montoya Arango, García Sánchez y Ospina Mesa, 2013); en el producto que siempre será un relato compartido; y en el contenido del mapa que siempre será de relevancia para la comunidad y por tanto favorecedor de transformaciones y procesos de emancipación (Ares y Risle, 2013).

Esta última afirmación nos sitúa en las relaciones que fácilmente se pueden encontrar entre la cartografía social y la EpDCG. Así, Leivas (2019) afirma que la cartografía social es un método capaz de abordar los problemas relacionados con el territorio y de promover el desarrollo de conocimientos, habilidades y actitudes para la ciudadanía global. Sin embargo, como sabemos, no existe una única forma de entender e incluso denominar a este campo de estudio. Las relaciones que encontramos entre EpDCG y la cartografía social requieren de una forma de entender la EpDCG cercanas a los enfoques críticos y a aquellas perspectivas que destacan la dimensión global (Quintano Nieto, Ortega Gaite, Tejedor Mardomingo y Ruiz Ruiz, 2019). Es decir, aquéllas que ponen el énfasis en la transformación social y el empoderamiento de una ciudadanía capacitada para analizar de forma crítica las dinámicas de poder, cuestionar sus propias concepciones y el pensamiento dominante, entender la corresponsabilidad y la interdependencia en el mundo global en el que vivimos y en las relaciones norte-sur, así como con capacidad para denunciar las desigualdades y actuar en pro de una justicia social. Desde estos enfoques se busca visibilizar y actuar contra las asimetrías globales existentes reflejadas en

la desigualdad entre países, el sometimiento de territorios y personas a los intereses del mercado, la pobreza sistémica, la dominación de los hombres sobre las mujeres, la 
persecución de la diferencia y del diferente, la sobreexplotación de recursos naturales, etc. (DePalma, 2019, p. 9).

Definiciones como la de Alejandra Boni (2017, en Leivas 2019) también reflejan estos enfoques. Ella afirma que la educación para la ciudadanía global es un proceso educativo que aspira a generar una conciencia crítica y transformadora, que conecta lo local con lo global, que tiene en cuenta las múltiples identidades que configuran al ser humano, en el que las personas se reconocen a sí mismas como parte de problemas pero también de las soluciones, y se ven como agentes de cambio que buscan promover la justicia social.

Por tanto, es desde esta perspectiva desde la que se puede presentar la cartografía social como idónea para acercarnos a los fines de una EpDCG crítica. Con ayuda de la cartografía social, se pueden crear estrategias que ayuden a reflexionar sobre las narrativas dominantes ya que, muchas de las propias prácticas de educación para el desarrollo, y otras prácticas educativas, reproducen representaciones del mundo hegemónicas, etnocéntricas, ahistóricas, despolitizadas, salvacionistas, no complicadas y paternalistas (Andreotti, 2014). Como decíamos antes, la construcción de mapas puede ayudarnos a desvelar nuestras propias posiciones y mostrar cómo cualquier interpretación se construye siempre social, cultural e históricamente y cómo todo mapa constituye una representación parcial de la realidad (Corbett y Donehower, 2017). La cartografía social puede ayudar a establecer esas situaciones de diálogo y discusión que autoras como Alyson Jenkins (2015) entienden como esenciales y eje de cualquier actividad pedagógica que quiera facilitar el aprendizaje en temas relacionados con la dimensión global. Tal y como explica la autora, las discusiones democráticas en el aula pueden ayudar a conectar las preocupaciones locales y globales, reflexionando sobre el proceso de convertirse en ciudadanía global con capacidad de transformación social.

\subsection{La colaboración entre actores y los proyectos de EpDCG}

La creación de redes de colaboración es el fin último de nuestro proyecto y una de las principales razones de escoger la cartografía social como método. El equipo de investigación lleva siete años trabajando en proyectos donde la colaboración entre agentes se ha convertido en una herramienta clave. El objetivo de esta colaboración parte de la necesidad de posibilitar la unión entre el mundo académico-investigador y el mundo de la acción, acercando la realidad y el análisis de la misma para tratar de dar respuesta a interrogantes clave y mejorar la práctica de la EpDCG, facilitando así la transferencia del conocimiento. Este objetivo ha prolongado la colaboración en el tiempo, llegando hasta la actualidad. 
A lo largo de estos años, y especialmente en un proyecto de investigación-acción participativa (IAP) que llevamos a cabo en un centro educativo gallego, se afianzó nuestro interés en la colaboración entre actores, demostrando que el promover una relación multi-actor, favorece análisis enriquecidos por la variedad de ópticas y perspectivas que se aportan. Este trabajo colaborativo nos ha permitido

ir apuntalando la relación entre el mundo académico y el mundo de la práctica para generar conocimiento de una manera sistemática y compartida (Cotelo Guerra, Pérez Crego, Iglesias Salvado y Pereiro González, 2019, pp. 261-262).

La investigación colaborativa entre docentes y profesionales de las ONGD ha sido estudiada entre otros, por Boni (2012), quien ha analizado los significados y las prácticas educativas que docentes de primaria, secundaria y universidad pueden realizar en conjunto con las ONGD para potenciar la ciudadanía global en el sistema educativo formal. Por su parte, Martins, Madeira y Gonçalves (2018), con un trabajo colaborativo desarrollado entre una institución de educación superior y dos organizaciones de la sociedad civil, presentan también resultados sobre el proceso colaborativo siguiendo tres ejes de análisis: la dinámica interpersonal de la colaboración; la tensión entre las pedagogías formales; y cuestiones relacionadas con el aprendizaje, conocimiento y autoconocimiento. Tal y como destacan Leivas y Boni (2017, p. 44):

es necesario generar espacios de diálogo entre diferentes actores dentro y fuera de la universidad, que permitan el diálogo y la comunicación reflexiva-crítica en la búsqueda de soluciones colectivas a las complejas problemáticas de la sociedad contemporánea.

Es en estos espacios en los que se pueden configurar propuestas donde la cultura escolar se entrelaza con la realidad social para poder crear conocimiento crítico y transformador, reforzando la capacidad social adaptativa del medio escolar de forma que responda adecuadamente a las posibilidades y desafíos de una sociedad caracterizada por estar en constante evolución (Cotelo-Guerra, et. al, 2019).

La colaboración entre actores es crucial en el mundo interdependiente, interconectado y global en el que nos encontramos, en el que

se hace evidente que trabajar con otros, desarrollar proyectos conjuntamente y compartir visiones, diagnóstico y teorías de cambio se convierte en algo fundamental si realmente se quiere tener un impacto significativo (Iglesias y Carreras, 2013, p. 11).

Para estos autores los factores clave del éxito de una colaboración significativa son: una visión compartida; respeto mutuo, tolerancia y confianza entre sus miembros; liderazgo comprometido; atención al proceso; equilibrio entre aportes y resultados; y un volumen adecuado de recursos. En esta línea, Ortega Carpio (2014), 
destaca las ventajas del trabajo conjunto entre la Universidad y las ONGD, para lo que es necesario:

que las actividades a desarrollar se dirijan a un objetivo conjunto, capaz de impulsar el compromiso colectivo; que las personas implicadas cuenten con el apoyo formal de sus entidades, por lo tanto, que exista una cierta estructura que sustente el proceso de colaboración; que las personas y entidades se perciban como iguales, con áreas de competencia que les son propias y en las que están legitimadas. La confianza es un aspecto crucial para una mayor eficacia en las acciones; y que las personas que colaboren tengan experiencia en los dominios de los otros y entiendan la cultura de ambas entidades (p. 8).

Se puede afirmar que la colaboración entre la universidad y distintas organizaciones sociales, establecida a partir de la responsabilidad compartida, permite, además del intercambio de experiencias o de herramientas para emprender proyectos (Morales y Martín, 2013), la consecución de nuevos aprendizajes, así como la mejora de la práctica comunitaria y de calidad de la investigación y la enseñanza (Castanheira, Barreto, Santos y Silva, 2016). En este sentido y, como resaltan Ortega Carpio, Cordón Pedregosa y Sianes (2013, p. 36), identificar, reunir y difundir buenas prácticas en el ámbito de la EpDCG es fundamental por los siguientes motivos:

Primero obtener una sistematización de Buenas Prácticas facilitaría la transmisión de conocimientos, al documentar y mejorar la transferibilidad entre actores y contextos diversos en un entorno tan atomizado como el español, con más de 70 universidades y centenas de ONGD trabajando en el ámbito de la ED. Segundo podría facilitar y promover la búsqueda de soluciones innovadoras, exitosas y sostenibles y tercero puede contribuir a aportar orientaciones excelentes para el desarrollo de iniciativas nuevas y, sobre todo, para la definición de estrategias y políticas en la materia.

El proyecto que estamos desarrollando en la actualidad se dirige, en este sentido, a visibilizar, compartir y motivar la puesta en marcha de proyectos de EpDCG.

\section{Método}

El método de investigación utilizado es la cartografía social y el instrumento de recogida de información la sala cartográfica. Una sala cartográfica es un espacio participativo en el que se reflexiona entorno a la creación de un mapa. En nuestro caso el mapa recoge experiencias de EpDCG que se llevan a cabo en Galicia en la educación reglada no Universitaria.

El proyecto de investigación tiene dos fases. La primera, que presentamos en este artículo, se llevó a cabo entre junio del 2018 y noviembre del 2019 , y su 
principal objetivo era la construcción del instrumento que permitirá mapear las experiencias de EpDCG en Galicia. La segunda fase, que se está desarrollando en la actualidad, tiene como objetivo el mapeo de experiencias de EpDCG en la provincia de A Coruña (Galicia).

Los objetivos específicos de la primera fase eran los siguientes:

1. Diseñar un instrumento que nos permitiese mapear, de forma colaborativa y con el fin de crear redes, las experiencias de EpDCG que se llevan a cabo en Galicia.

2. Realizar un estudio piloto para poner a prueba el instrumento diseñado (sala piloto).

Para conseguir estos objetivos se partió de varias premisas, siendo fieles a lo que constituye un proceso de cartografía social acorde a la vez con un proceso de IAP (Roura Expósito, Díaz Aguilar, Ruiz Blanch, Sánchez Carretero y Cortés Vázquez, 2018; Sírvent y Rigai, 2012):

- El proceso de investigación se debe entender de forma participativa, debiendo crearse dinámicas simétricas de colaboración en igualdad entre todos los participantes. El diseño y puesta en marcha del instrumento de investigación, con el que se pretende mapear experiencias de EpDCG y el propio proceso de creación de estos mapas, se concibe de forma participativa ya que lo que se busca es facilitar espacios de encuentro y colaboración.

- Este proceso de negociación de significados debe ser una constante a lo largo de toda la investigación. De forma dialogada se buscará establecer conexiones teoría-práctica, reflexionando sobre nuestras concepciones y nuestras experiencias. La construcción de las salas cartográficas se concibe como un proceso reflexivo de diálogo constante entre todos los participantes, todos aprendemos y enseñamos y los propios mapas creados se entienden como relatos compartidos.

- Estos relatos compartidos y el proceso participativo para llegar a ellos se constituyen en un conocimiento con intencionalidad transformadora, que debe tener relevancia para la comunidad, favoreciendo procesos de emancipación. El mapeo colaborativo ayuda a reflexionar sobre nuestras prácticas, lo que promueve su problematización y transformación. A su vez, se quiere ayudar a visibilizar las experiencias de EpDCG y crear redes de colaboración entre sus protagonistas.

Nos guiamos además por la definición de Fernando y Giraldo (2016, p. 256) de la cartografía social pedagógica (CSP): 
La CSP se puede entender como una estrategia de investigación y acompañamiento en la que, por medio de la acción colectiva, se lleva a los participantes a reflexionar sobre sus prácticas y comprensiones de una problemática común, mediante el levantamiento de un mapa (cartografía) en el que se evidencian las problemáticas que acontecen en dicho territorio; en este caso, los territorios relacionados con las tensiones de las prácticas de enseñanza y de aprendizaje. La finalidad de la CSP va más allá de la representación gráfica de los territorios donde acontecen las acciones educativas; implica vincular a los participantes en la posibilidad de transformar sus prácticas; se fortalecen lazos de cooperación y se fortifica el aprendizaje entre pares.

\section{I. Instrumento y materiales de recogida de datos}

La sala cartográfica fue el instrumento elegido para llevar a cabo el mapeo colaborativo de experiencias de EpDCG, es decir, para recoger información sobre las mismas y promover la reflexión y diálogo compartido. La decisión de utilizar la sala cartográfica fue fruto del proceso de formación en cartografía social ${ }^{5}$ llevado a cabo por el equipo inicial de investigación. A lo largo de este proceso, y en reuniones posteriores, se fue llegando a acuerdos sobre el contenido que llenaría la sala y la forma en la que los participantes interactuarían con el mismo, pudiendo modificarlo y mejorarlo. Las decisiones sobre el contenido de la sala estuvieron precedidas de intensos debates, reflexiones y negociaciones, buscando conceptualizaciones compartidas y deliberando sobre cuestiones como la siguientes: ¿qué tipo de información nos gustaría que se dibujase en el mapa?; ¿qué categorías podrían ayudar al participante a mapear sus experiencias?; ¿se debería definir previamente las categorías o dejar que surjan en el proceso?; ¿se deberían recoger únicamente experiencias de la última generación o también las enmarcadas en generaciones anteriores? Para responder a estas preguntas, además de los debates internos, también se contrastaron nuestras concepciones y conocimientos previos, con las aportaciones de estudios en los que se muestran distintos aspectos que abordan los proyectos de EpDCG (Aguado, 2011; Argibay y Celorio, 2005; Boni y López, 2015; Pérez Salgado, 2016). Así la sala beta (primera versión de la sala, ver Fig. 1 y 2) quedó configurada con los materiales y contenidos que presentamos en la Tabla 1.

\footnotetext{
${ }^{5}$ Este proceso de formación estuvo a cargo de consultores externos expertos en cartografía social y participativa, María Masaguer y Martín Barreiro.
} 


\section{TABLA I: Materiales y contenido sala beta}

\begin{tabular}{|c|c|c|}
\hline Material & Descripción & Ejemplo contenido \\
\hline $\begin{array}{l}\text { Ficha } \\
\text { Agente/ } \\
\text { Docente }\end{array}$ & $\begin{array}{l}\text { Datos relevantes sobre } \\
\text { la persona/s que pone } \\
\text { en marcha la experien- } \\
\text { cia de EpDCG. }\end{array}$ & $\begin{array}{l}\text { Centro de trabajo y localidad; Experiencias } \\
\text { desarrolladas (número, alumnado implicado, } \\
\text { entidades colaboradoras)... }\end{array}$ \\
\hline Ficha Centro & $\begin{array}{l}\text { Datos relevantes sobre } \\
\text { el lugar de trabajo del } \\
\text { o de la docente/agente. }\end{array}$ & $\begin{array}{l}\text { Nombre del centro; Localización; Tipo; Etapas } \\
\text { educativas; Nº Profesorado; No Alumnado. }\end{array}$ \\
\hline $\begin{array}{l}\text { Ficha } \\
\text { Experiencia }\end{array}$ & $\begin{array}{l}\text { Datos descriptivos de la } \\
\text { experiencia. }\end{array}$ & $\begin{array}{l}\text { Nombre de la experiencia; Quien promueve; } \\
\text { Desde cuándo;Duración; Ámbito de aplicación; } \\
\text { Etapa educativa; Área/materia; Formato; } \\
\text { Materiales;... }\end{array}$ \\
\hline \multirow[t]{2}{*}{$\begin{array}{l}\text { Panel } \\
\text { Objetivos/ } \\
\text { Dimensiones }\end{array}$} & \multirow{2}{*}{$\begin{array}{l}\text { Objetivos y dimensio- } \\
\text { nes de la EpDCG en } \\
\text { las que encuadraría la } \\
\text { experiencia. }\end{array}$} & $\begin{array}{l}\text { Objetivos: Recaudar; Visibilizar una realidad } \\
\text { problemática; Conocer/comprender las causas } \\
\text { de una realidad problemática; Acciones de in- } \\
\text { cidencia política; Cuestionamiento global-local, } \\
\text { corresponsabilidad, interdependencia... }\end{array}$ \\
\hline & & $\begin{array}{l}\text { Dimensiones: sensibilización; formación; } \\
\text { incidencia política; movilización social; } \\
\text { investigación. }\end{array}$ \\
\hline $\begin{array}{l}\text { Panel } \\
\text { Temáticas }\end{array}$ & $\begin{array}{l}\text { Temáticas trabajadas en } \\
\text { la experiencia. }\end{array}$ & $\begin{array}{l}\text { Ciudadanía digital, comercio justo, correspon- } \\
\text { sabilidad, cultura de paz, derechos humanos, } \\
\text { diversidad funcional, feminismo, globalización, } \\
\text { interculturalidad, interdependencia, justicia so- } \\
\text { cial, medioambiente, pobreza, sostenibilidad,... }\end{array}$ \\
\hline $\begin{array}{l}\text { Panel } \\
\text { Competencias }\end{array}$ & $\begin{array}{l}\text { Competencias trabaja- } \\
\text { das con la experiencia. }\end{array}$ & Aprender a saber, a hacer, a ser, a convivir. \\
\hline $\begin{array}{l}\text { Panel } \\
\text { Metodologías }\end{array}$ & $\begin{array}{l}\text { Metodologías y técnicas } \\
\text { utilizadas. }\end{array}$ & $\begin{array}{l}\text { Aprendizaje basado en problemas; Aprendi- } \\
\text { zaje basado en proyectos; Estudio de casos; } \\
\text { Aprendizaje-servicio, Aprendizaje cooperati- } \\
\text { vo; Prácticas TIC... }\end{array}$ \\
\hline $\begin{array}{l}\text { Panel } \\
\text { Evaluación }\end{array}$ & Aspectos evaluados. & $\begin{array}{l}\text { De los aprendizajes; de la experiencia; del } \\
\text { propio agente. }\end{array}$ \\
\hline Entrevista & $\begin{array}{l}\text { Entrevista estructurada } \\
\text { grabada en vídeo. }\end{array}$ & $\begin{array}{l}\text { Qué te motivó a realizar la experiencia, qué } \\
\text { impacto tuvo, por quécrees que mereció la pena. }\end{array}$ \\
\hline Mapa & $\begin{array}{l}\text { Mapa político de } \\
\text { Galicia. }\end{array}$ & \\
\hline
\end{tabular}




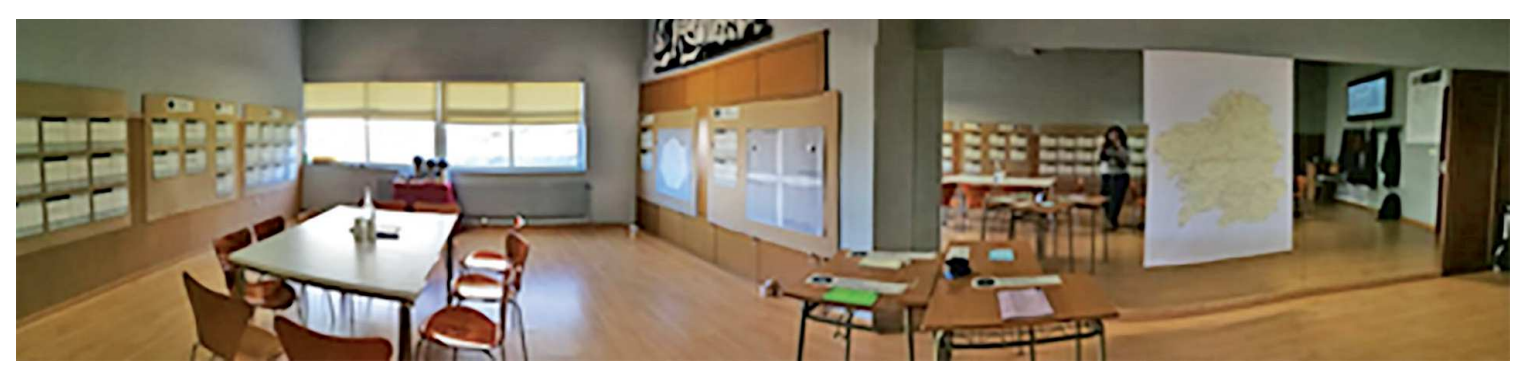

Fig. 1 Panorámica sala beta antes de su comienzo.

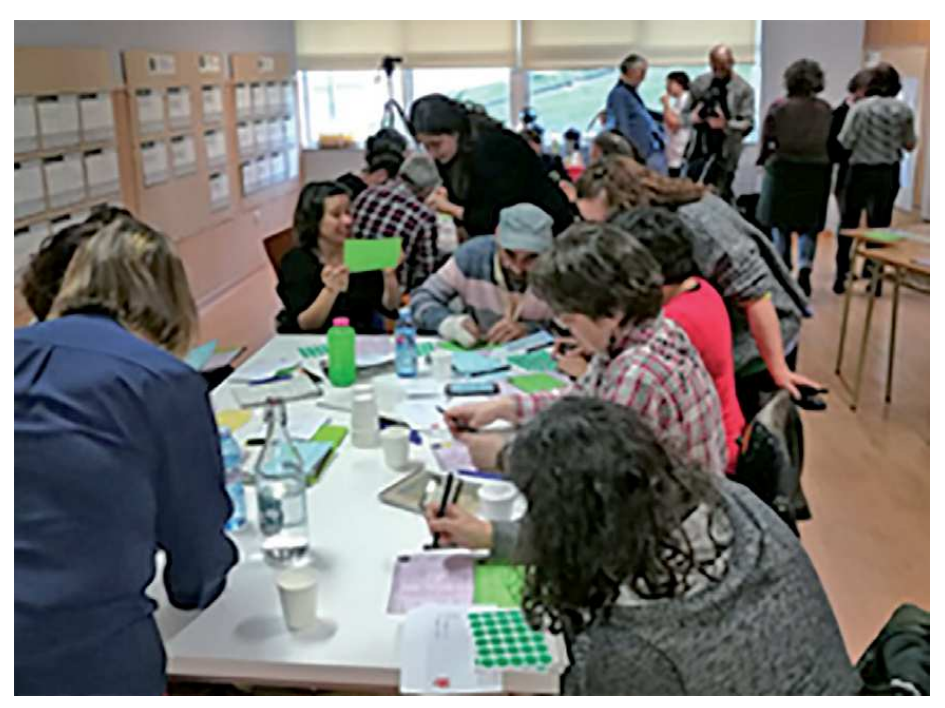

Fig. 2 Sala beta durante su desarrollo.

Otra decisión relevante tuvo que ver con los participantes de la sala beta. Tras varias reuniones y negociaciones se optó por invitar a docentes con experiencia en proyectos de EpDCG y agentes de entidades que trabajan en este campo. Ellos y ellas serían los participantes clave que pasarían a formar parte del equipo de investigación, colaborando en la construcción del instrumento, a la vez que mapeaban sus experiencias. Así, esta sala se llevó a cabo en la Facultad de Ciencias de la Educación (Universidad de A Coruña) el mes de marzo del 2019, participando veintiocho personas, diez miembros de ONGs, cinco docentes de centros educativos no universitarios, tres personas que respondían a los dos perfiles anteriores, dos profesores universitarios y dos evaluadoras externas pertenecientes a la consultora que evalúa la actividad de SIG, además de seis personas del grupo inicial de investigación.

La sala se desarrolló en cuatro momentos: presentación de la sesión; codificación y cumplimentación de las fichas; interacción con los paneles y el mapa; valoración 
grupal final de los materiales elaborados (fichas y paneles), así como del funcionamiento de la sala, a través de un grupo focal.

\subsection{Análisis de datos derivados del desarrollo de la sala beta}

Dado que el objeto de esta fase era la construcción y validación del instrumento para mapear experiencias se realizó un análisis de los datos obtenidos en el desarrollo de la versión beta de la sala cartográfica, mediante un proceso inductivo de categorización (Miles, Huberman y Saldaña, 2014; Silverman, 2012) de la siguiente información:

- la derivada de la cumplimentación de los materiales de la propia sala, léase fichas, paneles y mapa (código RFPSB);

- los comentarios que podían realizar en post-it respecto de cada panel (código CPSB);

- la generada en el grupo focal de valoración del instrumento (código GFSB);

- y las observaciones del equipo de investigación, registradas en entrevistas individuales a cada integrante, tras finalizar la sesión (código EISB).

El grupo focal y las entrevistas individuales a las integrantes del grupo de investigación fueron grabadas en audio y transcritas. Las categorías y subcategorías de análisis fueron las siguientes: material (fichas, paneles, mapa); dinámica (tiempo, comprensión, reflexión, codificación); sala (utilidad, interés).

El análisis de esta información, en sendas reuniones, permitió mejorar el instrumento de cara a la realización de la sala piloto, tomando de nuevo decisiones sobre su contenido y funcionamiento. Esta segunda sala tenía como objetivo pilotar el instrumento y a la vez que se mapeaban experiencias de EpDCG que se llevan a cabo en centros educativos de la ciudad de A Coruña y su área metropolitana. Se llevó a cabo en el Centro de Formación y Recursos de A Coruña en el mes de noviembre del 2019 (ver Fig. 3). En ella participaron nueve personas, dos docentes, dos miembros de ONG, una persona que respondía a ambos perfiles y cuatro personas del equipo inicial de investigación. 


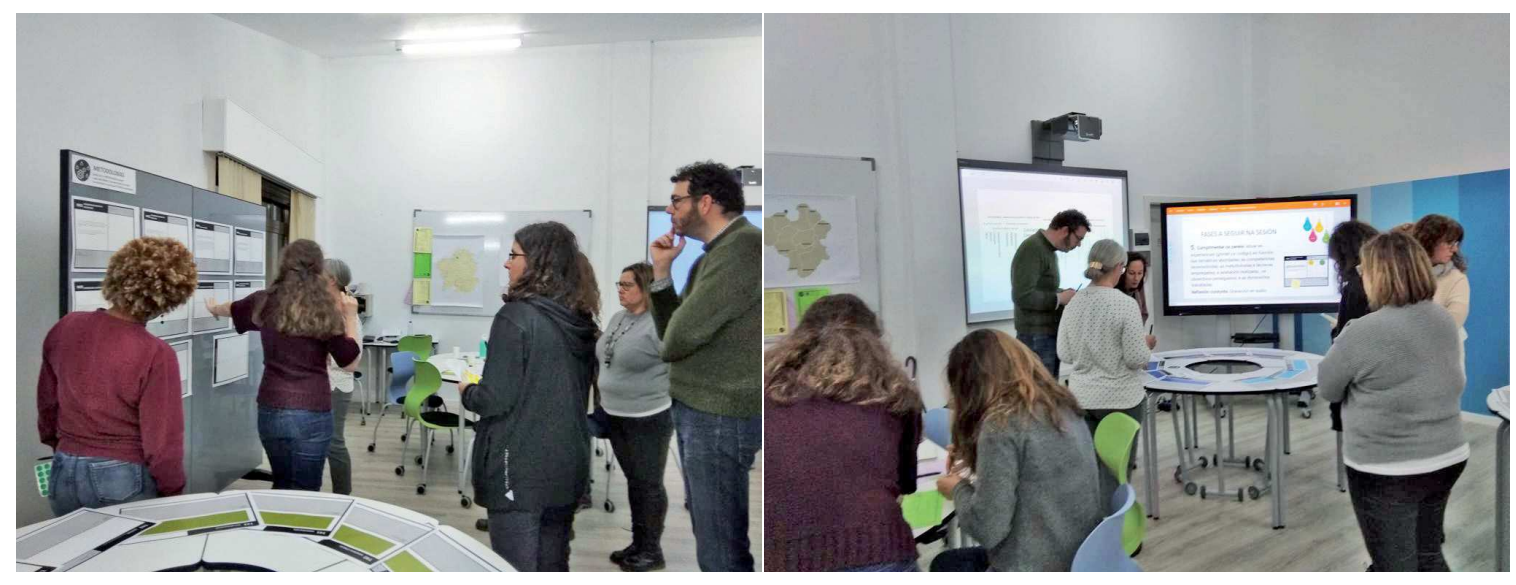

Fig 3. Sala piloto durante su desarrollo.

Tras esta sala se llevaron a cabo nuevamente procesos de análisis del contenido y funcionamiento, para poder seguir mejorando el instrumento. En este caso, además de los comentarios en paneles y de las respuestas en fichas, paneles y mapa, se realizaron dos grupos focales con los participantes (uno inicial y otro final), grabados en audio, transcritos y analizados (códigos GFISP y GFFSP). El contenido de la sala piloto difirió en diversos aspectos del de la sala beta, como se mostrará en los resultados.

Derivado del desarrollo de ambas salas se obtuvo un primer mapeo de experiencias de EpDCG. El análisis de estos datos no formaba parte de los objetivos de esta fase del proyecto, pero se realizó igualmente una primera caracterización, lo que permitió extraer unas reflexiones preliminares que presentamos en los resultados.

\section{Resultados}

\section{I. Sobre el instrumento: contenido y funcionamiento de la sala cartográfica}

Comenzando por los resultados referidos a la construcción y validación del instrumento, derivados de la realización de la versión beta de la sala cartográfica y del análisis de los datos recogidos, destacamos los siguientes:

- Se sugirieron mejoras en las fichas y paneles y se observó la necesidad de simplificar la codificación y reducir la información requerida (en este sentido, 
se fusionaron las fichas de centros educativos y experiencias). Los paneles más comentados fueron el de temáticas (se decidió su organización en categorías, sin renunciar a presentar todas las elaboradas, revisando e incluso incluyendo otras nuevas); y el de competencias (se incluyeron, como se sugería, las recogidas en la actual Ley de educación española ${ }^{6}$, manteniendo los cuatro pilares de Delors (1996), como oportunidad de matizar la información requerida). (Ver Tabla 2).

\section{TABLA 2. Ejemplos de mejoras propuestas en la sala beta}

\begin{tabular}{|c|c|c|}
\hline Material & Código & Ejemplos de datos \\
\hline \multirow{3}{*}{ FICHAS } & GFSB & $\begin{array}{l}\text { Informante 4_Docente: "Falta añadir quién se beneficia de la } \\
\text { experiencia". }\end{array}$ \\
\hline & RFPSB & $\begin{array}{l}\text { Se observan diferencias significativas entre las fichas cubiertas } \\
\text { por docentes y por agentes de ONG, por lo que se requiere hacer } \\
\text { modificaciones en las fichas dirigidas a agentes para adaptarlas } \\
\text { a su realidad. }\end{array}$ \\
\hline & GFSB & $\begin{array}{l}\text { Informante 13_Agente: "Es difícil cubrir la ficha de centro porque } \\
\text { trabajamos la misma experiencia en centros diferentes". }\end{array}$ \\
\hline \multirow{4}{*}{ PANELES } & CPSB & $\begin{array}{l}\text { Informante 6_Docente: "No veo la diferencia entre consumo res- } \\
\text { ponsable y comercio justo". }\end{array}$ \\
\hline & EISB & $\begin{array}{l}\text { Investigadora 4: "Parece necesario agrupar las temáticas bajo } \\
\text { categorías más globales". }\end{array}$ \\
\hline & GFSB & $\begin{array}{l}\text { Informante 3_Docente: "Es preferible incluir las competencias } \\
\text { incluidas en la actual Ley de educación en lugar de las de Delors } \\
\text { porque estas no responden a vocabulario habitual del profesorado, } \\
\text { parecen más orientadas al ámbito de la educación social". }\end{array}$ \\
\hline & CPSB & $\begin{array}{l}\text { Informante 1_Docente: Añade en comentarios la metodología } \\
\text { "Visual thinking". }\end{array}$ \\
\hline
\end{tabular}

- En cuanto a la dinámica, se puso de manifiesto la necesidad de comenzar haciendo una presentación resumida de todo el proceso de desarrollo de la sala para, a continuación, ir guiando de manera pausada y conjunta cada una de las fases de la misma. Comenzando por la cumplimentación de las fichas para

\footnotetext{
${ }^{6}$ La Ley Orgánica 8/2013, de 9 de diciembre, para la mejora de la calidad educativa establece siete competencias clave: comunicación lingüística; matemática y básicas en ciencia y tecnología; digital; aprender a aprender; sociales y cívicas; sentido de la iniciativa y espíritu emprendedor; conciencia y expresiones culturales.
} 
continuar con la interacción con el mapa y los paneles. Asimismo, se destacó la importancia de dedicar tiempo a compartir las experiencias de cada participante, animando la reflexión durante el recorrido de la sala, así como el diálogo y la negociación de significados.

- Hace falta familiarizarse con el material porque al principio es mucha información (GFSB; Informante12_Agente)

- Explicar tarea por tarea, presentar cada ficha por separado y no distribuir nuevas fichas hasta que se cubran las anteriores" (EISB; Investigadora 3)

- Tengo la sensación de que la gente trabajó demasiado rápido, sin detenerse lo suficiente para reflexionar (EISB; Investigadora 5)

Todas estas modificaciones indicaban claramente que la sala cartográfica funcionaría mejor con menos participantes y más tiempo, como se pudo demostrar en la sala piloto

- Quizás es preferible menos participantes para fomentar el diálogo y debate entre todos (EISB; Investigadora 5)

Respecto de la sala piloto, el análisis de la información recogida apunta que los materiales elaborados son adecuados, solo se plantea una nueva modificación en el panel de competencias, resultando esta dimensión todavía demasiado compleja. Así, se propone como alternativa utilizar únicamente las competencias recogidas en la Ley de educación vigente como eje de análisis de las experiencias.

\subsection{Del primer mapeo de experiencias en las salas beta y piloto}

En el desarrollo de las dos salas cartográficas, los participantes tuvieron la oportunidad de compartir relatos sobre las experiencias que habían desarrollado en su trayectoria profesional. En la Fig. 4 podemos ver las geolocalizaciones de las experiencias mapeadas de forma analógica y digital y en la Tabla 3 se muestran ejemplos de experiencias definidas en base a la información de algunos de los paneles ${ }^{7}$.

\footnotetext{
${ }^{7}$ Algunas de estas experiencias están geolocalizadas en varios lugares por haberse llevado a cabo en más de un centro.
} 


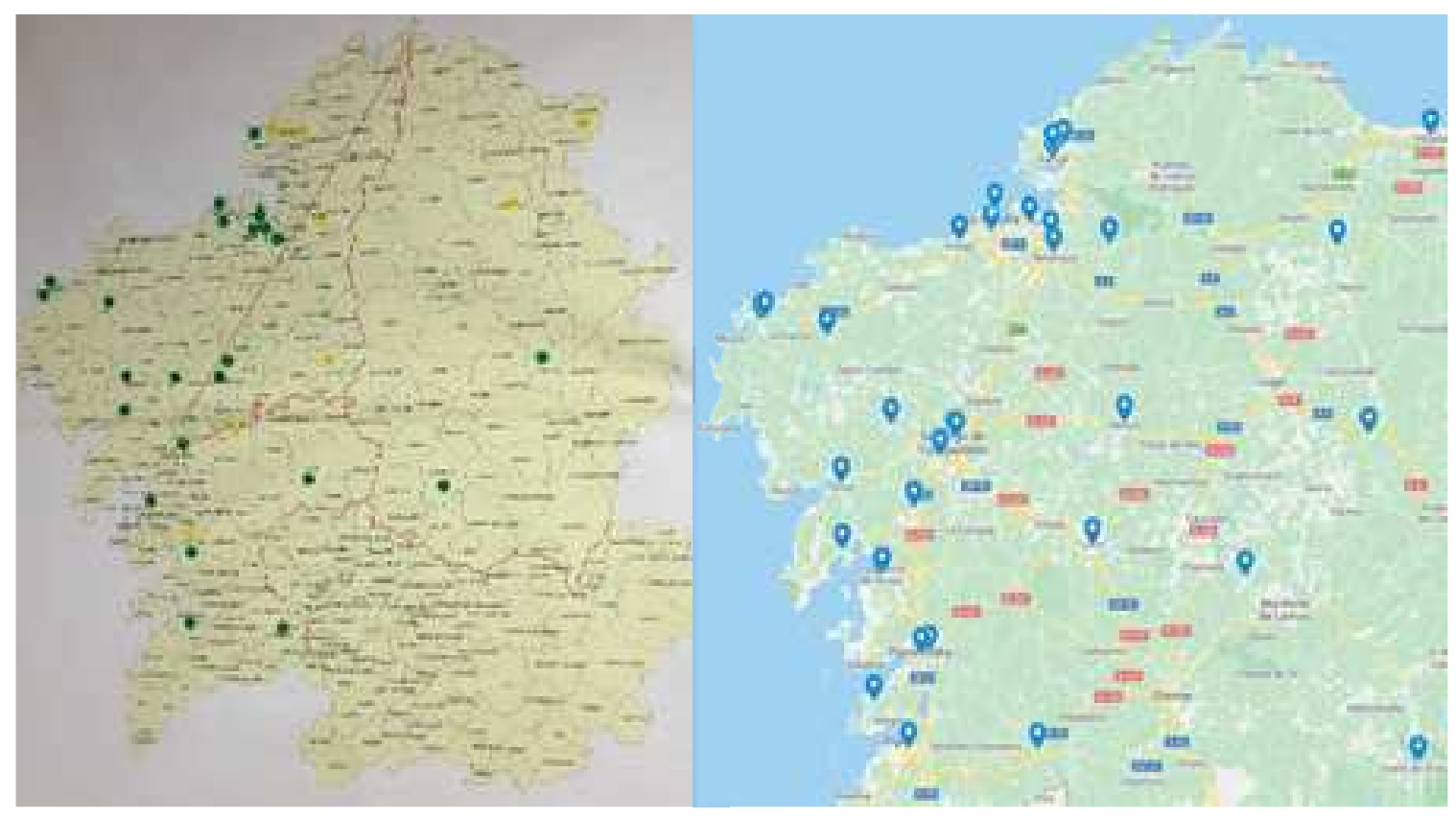

Fig. 4. Mapas analógico y digital geolocalización experiencias.

Lo primero que destaca es la predominancia de experiencias desarrolladas en la etapa secundaria obligatoria o postobligatoria, siendo necesario hacer mención también a las experiencias a nivel de centro o inter-nivel.

De las veintisiete experiencias mapeadas es interesante observar cómo ninguna se centra en el objetivo 1) de recaudar fondos o bienes ante una situación de emergencia, muy superado en la visión actual de la EpDCG. Por contra, las iniciativas se centran fundamentalmente en objetivos de reflexión crítica (conocer y comprender las causas de una realidad problemática, atendiendo a la dimensión estructural; y análisis crítico de la interdependencia Norte-Sur). Asimismo, a pesar de que hay cinco experiencias dirigidas a la incidencia política y movilización social, ninguna escogió el objetivo 6) de alcanzar cambios a nivel político, denunciar injusticias o impugnar decisiones políticas.

Vemos cómo también predomina el abordaje de la dimensión de sensibilización (nueve en total), sobre temáticas tan diversas como sostenibilidad, cultura de paz, colectivos vulnerables, interculturalidad, hábitat... En relación con esto, otro resultado interesante es que la EpDCG admite numerosas temáticas, como también metodologías, tal como se puede observar en la Tabla 3. 
TABLA 3. Experiencias mapeadas

\begin{tabular}{|c|c|c|c|c|c|}
\hline Experiencias & Etapa & $\begin{array}{l}\text { Temática } \\
\text { Principal }\end{array}$ & Objetivo & Metodología & Dimensión \\
\hline Tutoría entre iguales & ESO & Cultura de paz & $\begin{array}{l}\text { Cuestionamiento glo- } \\
\text { bal, corresponsabili- } \\
\text { dad, interdependencia } \\
\text { (O7) }\end{array}$ & $\begin{array}{l}\text { Aprendizaje- } \\
\text { servicio (APS) }\end{array}$ & Investigación \\
\hline lgualdad mates & ESO & Coeducación & $\begin{array}{l}\text { Visibilizar una rea- } \\
\text { lidad problemática } \\
\text { (O2) }\end{array}$ & $\begin{array}{l}\text { Aprendizaje basado } \\
\text { en proyectos }\end{array}$ & Investigación \\
\hline $\begin{array}{l}\text { Talleres } \\
\text { emprendimiento } \\
\text { solidario }\end{array}$ & $\begin{array}{l}\text { ESO, FP } \\
\text { Básica, } \\
\text { Bachillerato }\end{array}$ & Economía social & $\begin{array}{l}\text { Impulsar posibles so- } \\
\text { luciones poniendo en } \\
\text { práctica acciones con- } \\
\text { cretas (O5) }\end{array}$ & $\begin{array}{l}\text { Aprendizaje } \\
\text { cooperativa }\end{array}$ & Investigación \\
\hline No te consumas & $\begin{array}{l}\text { ESO, } \\
\text { Bachillerato }\end{array}$ & Comercio Justo & $\begin{array}{l}\text { Conocer/ comprender } \\
\text { las causas de una } \\
\text { realidad problemática } \\
\text { (O3) }\end{array}$ & $\begin{array}{l}\text { Aprendizaje } \\
\text { cooperativa }\end{array}$ & $\begin{array}{l}\text { Incidencia } \\
\text { política y } \\
\text { movilización } \\
\text { social }\end{array}$ \\
\hline Monfero solidario & $\begin{array}{l}\text { Infantil, } \\
\text { Primaria, ESO }\end{array}$ & $\begin{array}{l}\text { Ética y valores } \\
\text { solidarios }\end{array}$ & O7 & $\begin{array}{l}\text { Comunidades de } \\
\text { aprendizaje }\end{array}$ & Formación \\
\hline Kaya clínica & Universidad & Territorio & $\begin{array}{l}\text { Buscar soluciones, } \\
\text { propuestas de cam- } \\
\text { bio o alternativas a las } \\
\text { causas identificadas } \\
\text { de realidades proble- } \\
\text { máticas (O4) }\end{array}$ & APS & Incidencia... \\
\hline $\begin{array}{l}\text { El Tesoro de } \\
\text { Acahualinca }\end{array}$ & ESO & $\begin{array}{l}\text { Colectivos } \\
\text { vulnerables }\end{array}$ & О3 & $\begin{array}{l}\text { Comunidades de } \\
\text { aprendizaje }\end{array}$ & Sensibilización \\
\hline
\end{tabular}




\begin{tabular}{|c|c|c|c|c|c|}
\hline Experiencias & Etapa & $\begin{array}{l}\text { Temática } \\
\text { Principal }\end{array}$ & Objetivo & Metodología & Dimensión \\
\hline Elmundo que queremos & ESO & $\begin{array}{l}\text { Consumo respon- } \\
\text { sable }\end{array}$ & O3 & $\begin{array}{l}\text { Aprendizaje } \\
\text { colaborativa }\end{array}$ & Formación \\
\hline Vaya tela & ESO & $\begin{array}{l}\text { Consumo respon- } \\
\text { sable }\end{array}$ & O3 & $\begin{array}{l}\text { Aprendizaje } \\
\text { colaborativa }\end{array}$ & Formación \\
\hline $\begin{array}{l}\text { Horizonte } 2030 \text {-acer- } \\
\text { camiento a la realidad }\end{array}$ & FP Superior & Economía social & O7 & Estudio de caso & Formación \\
\hline $\begin{array}{l}\text { Cooperación al desa- } \\
\text { rrollo en ingeniería civil }\end{array}$ & Universidad & Sostenibilidad & O5 & $\begin{array}{l}\text { Aprendizaje basado } \\
\text { en proyectos }\end{array}$ & Sensibilización \\
\hline $\begin{array}{l}\text { Derecho al hábitat me- } \\
\text { diante la participación } \\
\text { social IES Zalaeta }\end{array}$ & ESO & $\begin{array}{l}\text { Derechos } \\
\text { humanos }\end{array}$ & O7 & $\begin{array}{l}\text { Aprendizaje } \\
\text { colaborativa }\end{array}$ & - \\
\hline $\begin{array}{l}\text { Difusión derecho hábi- } \\
\text { tat y los componentes } \\
\text { degénero, multicultura- } \\
\text { lidad y medioambiente }\end{array}$ & ESO & Territorio & O7 & $\begin{array}{l}\text { Aprendizaje basado } \\
\text { en proyectos }\end{array}$ & - \\
\hline $\begin{array}{l}\text { Acción difusión dere- } \\
\text { cho al hábitat en cola- } \\
\text { boración con proyecto } \\
\text { Terra }\end{array}$ & $\begin{array}{l}\text { Primaria } \\
\text { ESO }\end{array}$ & $\begin{array}{l}\text { Derechos } \\
\text { humanos }\end{array}$ & O7 & $\begin{array}{l}\text { Aprendizaje } \\
\text { colaborativa }\end{array}$ & - \\
\hline Conectando mundos & $\begin{array}{l}\text { Infantil, } \\
\text { Primaria, ESO, } \\
\text { Bachillerato }\end{array}$ & $\begin{array}{l}\text { Derechos sociales } \\
\text { Corresponsabi- } \\
\text { lidad }\end{array}$ & O3 & $\begin{array}{l}\text { Comunidades de } \\
\text { aprendizaje }\end{array}$ & Incidencia... \\
\hline $\begin{array}{l}\text { El mundo que queremos } \\
2018-19\end{array}$ & $\begin{array}{l}\text { ESO, Bachillera- } \\
\text { toFP Básica }\end{array}$ & $\begin{array}{l}\text { Corresponsabi- } \\
\text { lidad }\end{array}$ & O3 & $\begin{array}{l}\text { Aprendizaje basado } \\
\text { en proyectos }\end{array}$ & Formación \\
\hline
\end{tabular}




\begin{tabular}{|c|c|c|c|c|c|}
\hline Experiencias & Etapa & $\begin{array}{l}\text { Temática } \\
\text { Principal }\end{array}$ & Objetivo & Metodología & Dimensión \\
\hline $\begin{array}{l}\text { Escuelas sin racismo } \\
(2018-2019)\end{array}$ & $\begin{array}{l}\text { Infantil, Primaria, } \\
\text { ESO, FP Básica, } \\
\text { Universidad }\end{array}$ & Cultura de Paz & O3 & $\begin{array}{l}\text { Aprendizaje } \\
\text { cooperativa }\end{array}$ & $\begin{array}{l}\text { Sensibilización } \\
\text { e Incidencia... }\end{array}$ \\
\hline Epdlab & ESO & $\begin{array}{l}\text { Corresponsabi- } \\
\text { lidad }\end{array}$ & O3 & $\begin{array}{l}\text { Aprendizaje } \\
\text { colaborativo }\end{array}$ & Formación \\
\hline $\begin{array}{l}\text { Ayuntamientos coru- } \\
\text { ñeses con el comercio } \\
\text { justo }\end{array}$ & $\begin{array}{l}\text { Primaria } \\
\text { ESO }\end{array}$ & Comercio Justo & O3 & Estudio de caso & Sensibilización \\
\hline Barrios abiertos & $\begin{array}{l}\text { ESO } \\
\text { Bachillerato }\end{array}$ & Interculturalidad & O3 & $\begin{array}{l}\text { Prácticas TICy medios } \\
\text { de comunicación }\end{array}$ & Sensibilización \\
\hline Bicicletas solidarias & FP Básica & Medio Ambiente & O5 & $\begin{array}{l}\text { Aprendizaje } \\
\text { cooperativa }\end{array}$ & Formación \\
\hline $\begin{array}{l}\text { Cargados de futuro- } \\
\text { Gala benéfica }\end{array}$ & $\begin{array}{l}\text { ESO } \\
\text { Bachillerato }\end{array}$ & $\begin{array}{l}\text { Derechos } \\
\text { humanos }\end{array}$ & $\mathrm{O} 2$ & $\begin{array}{l}\text { Aprendizaje } \\
\text { colaborativa }\end{array}$ & Sensibilización \\
\hline $\begin{array}{l}\text { El derecho al hábitat } \\
\text { de Catabois }\end{array}$ & ESO & Territorio-Hábitat & O3 & $\begin{array}{l}\text { Aprendizaje } \\
\text { colaborativa }\end{array}$ & Sensibilización \\
\hline $\begin{array}{l}\text { En navidad; no te } \\
\text { consumas }\end{array}$ & Primaria & $\begin{array}{l}\text { Consumo respon- } \\
\text { sable }\end{array}$ & O5 & $\begin{array}{l}\text { Aprendizaje basado } \\
\text { en problemas }\end{array}$ & Sensibilización \\
\hline $\begin{array}{l}\text { Participar para } \\
\text { transformar }\end{array}$ & ESO & $\begin{array}{l}\text { Diversidad } \\
\text { funcional }\end{array}$ & O3 & APS & Incidencia... \\
\hline $\begin{array}{l}\text { Educar para encontrar- } \\
\text { nos; los cuentos como } \\
\text { herramienta para el } \\
\text { trabajo intercultural }\end{array}$ & Primaria & Interculturalidad & O3 & Otros & Sensibilización \\
\hline
\end{tabular}




\subsection{Del objetivo de promover la reflexión y crear redes de colaboración}

Además de identificar y mapear experiencias, la sala cartográfica tiene como objetivo promover la reflexión sobre EpDCG y tejer redes de colaboración. En este sentido, el hecho de compartir los relatos contribuyó al empoderamiento de los participantes. Entre estos relatos nos gustaría destacar dos de la sala piloto. Uno de ellos fue narrado por una persona con una larga trayectoria en el campo de la EpDCG, que responde, además, al perfil de docente y agente, al ser miembro de una ONG. El segundo es el de un docente que llegó a la sala sin tener claro si sus proyectos eran EpDCG:

...estoy hablando de un centro en un contexto de asentamientos precarios, donde el $60 \%$ de los estudiantes son de etnia gitana (...) El proyecto es introducir el derecho al hábitat en este contexto. Hicimos un mapeo muy simple con el tema "lo que me gusta y no me gusta del hábitat en el que vivo". Fue un pequeño mapeo sensible, para hacerlos visibles porque muchos no se conocían... y trabajamos el tema de la pobreza, analizaron qué necesidades se satisfacían, cuáles no, cuáles eran esas necesidades... Mi intención era motivar también con el profesorado porque son agentes multiplicadores... (GFISP; Informante19_Docente; 00:10:09-9).

... el proyecto se llamó "Bicicletas solidarias", partió de la dirección del centro y se llevó a cabo sobre todo en mi aula del grupo de FP básico. Los estudiantes, miembros de su familia, y otras personas de un instituto cercano aportaron bicicletas... nosotros las restauramos, modificamos, mejoramos y pusimos en funcionamiento y luego se las entregamos a una ONG (GFISP; Informante17_Docente; 00:40:33).

Compartir las experiencias, narrarlas e intentar situarlas en los paneles ayudó a los participantes a reflexionar sobre las mismas, a encontrar puntos de encuentro e imaginar posibles colaboraciones, como se muestra en este fragmento del diálogo entre estos dos participantes:

...noté ciertas carencias... Para organizar un proyecto de este tipo tiene que haber una estructura, un líder del proyecto, un seguimiento, responsabilidad... no puede basarse solo en el querer hacer de algunas personas, así no se puede sostener (GFISP; Informante17_Docente_00:49:10-2).

...pienso un poco como tú, creo que si no son actos resistentes desde la acción y con convicción no funcionan. Cuando lo contabas pensaba, bueno presentaron un proyecto que tenía una visión casi de aprendizaje-servicio, que quizá no relacionaron directamente con más áreas del curriculum,... pero digo guau!, menudo filón!, y especialmente porque cuando llegaste dijiste "no sé si esto que hice vale..." pero si incluso se podía hablar sobre los sistemas de movilidad que nos están inculcando... (GFISP; Informante19_Docente; 00:50:24-3). 
...bueno sí, es que incluso tuvo impactos muy positivos porque el alumnado se motivó con el tema de las bicicletas y empezaron a venir al instituto en bicicleta (GFISP; Informante17_Docente; 00:51:08-3).

Los participantes de ambas salas coincidieron en su valoración global, afirmando que había sido una experiencia enriquecedora, a pesar de que algunos se sintieron abrumados al principio por la cantidad de materiales que componían la sala:

Entrar aquí era para echarse para atrás... Es cierto que es mucha información y que había que leer, pero bueno creo que es positivo en el sentido de que hace reflexionar, regurgitar, rumiar toda esa experiencia. Aunque fuera a bote pronto, creo que los resultados sí facilitan y orientan y clarifican mucho lo hecho y lo que queda por hacer (GFISP; Informante6_Docente; 00:03-43).

\section{Discusión}

En el desarrollo de la primera fase de la investigación, que se presenta en el artículo, se han alcanzado de forma satisfactoria los objetivos formulados. Se comenzó el proyecto llevando a cabo un proceso formativo que fue determinante para decantarnos por la sala cartográfica como instrumento para el mapeo de experiencias de EpDCG, y para decidir de forma conjunta y dialogada el diseño y contenido de la misma, buscando en todo momento que se favoreciese la colaboración entre actores y la creación de redes, como fines últimos de la investigación.

Si bien es cierto que los materiales de las salas fueron diseñados por el equipo de investigación, el desarrollo de la versión beta y piloto constituyeron espacios imprescindibles para problematizar y repensar el contenido y funcionamiento de la propia sala. Tal como se mostró en los resultados, la reflexión compartida conllevó cambios significativos en su diseño. Esto solo sería posible si se lograba crear un espacio que motivase a los participantes a contar y compartir creando relatos conjuntos que favoreciesen transformaciones y procesos de emancipación (Ares y Risle, 2013; Fendler, 2013). Se puede afirmar que cumplimos con los aspectos definitorios de la cartografía social como proceso de creación colaborativa, facilitando espacios de encuentro y mediación de significados (Montoya Arango, García Sánchez y Ospina Mesa, 2013) sobre lo que supone una experiencia educativa de EpDCG, promoviendo la (auto)reflexión (Andreoti, 2014; Leivas, 2019), permitiendo deconstruir nuestras propias (pre)concepciones y representar un contenido con relevancia para la comunidad y con potencialidad transformadora y emancipadora (Ares y Risle, 2013). Se partía de lo local y particular (la 
experiencia concreta de cada participante) para, a través del diálogo y reflexión, consensuar un relato compartido, en el que las diferentes experiencias quedan interconectadas, estableciendo redes de conocimiento y relación entre agentes, docentes e investigadoras.

La realización de ambas salas (beta y piloto) trajo consigo un primer mapeo de experiencias. Aunque el análisis de estos resultados no era parte de los objetivos de esta fase del proyecto, la caracterización de las iniciativas de EpDCG ya mapeadas empieza a arrojar interesantes evidencias sobre el tipo de proyectos que se llevan a cabo. En este sentido, cabe destacar la predominancia de un enfoque crítico orientado a cuestionar y generar conciencias que analicen el estado de las cosas, examinando las causas estructurales de una realidad problemática. Cabía esperar que esto fuese así, puesto que la mayor parte de las experiencias mapeadas lo han sido de manos de personas expertas invitados intencionalmente a participar en la sala beta, pero también es cierto que partir de una versión del mapa rico en experiencias que reflejan un enfoque crítico incrementa la potencialidad transformadora del mismo.

En esta misma línea fue reconfortante encontrar experiencias que se desarrollen más allá del aula, involucrando a varias etapas educativas, habiendo testimonios incluso de experiencias a nivel de centro. Localizar y publicitar este tipo de iniciativas contribuirá, sin lugar a dudas, a superar ese enfoque reduccionista sobre la presencia de la EpDCG en la formación reglada no universitaria que venimos detectando en nuestra trayectoria investigadora (DePalma, 2019). Por su parte, el predominio de la dimensión de sensibilización nos mantiene alerta en la necesidad de incidir en la formación como aliada hacia el cambio y la emancipación.

En el desarrollo de las dos sesiones, los participantes tuvieron la oportunidad de reconocer sus prácticas y proyectos, a la vez que se favorecía la reflexión sobre las mismas y se compartían las experiencias de otros, creándose esos espacios de diálogo y comunicación reflexiva-crítica tan necesarios (Leivas y Boni, 2017). Este reconocimiento y el hecho de compartirlo puede convertirse en un estímulo para poner en marcha nuevas iniciativas de colaboración entre actores, tanto para aquellos que ya tienen cierta trayectoria en el campo, como para otros que podrían animarse a comenzar proyectos de EpDCG. Los resultados de las salas y la satisfacción mostrada por sus participantes revelaron esta gran potencialidad como espacio de encuentro y colaboración.

Respecto de las limitaciones, se evidenció que la sala funcionaba mejor con un número reducido de participantes. Esto implica que en cada sesión se mapearía 
un número reducido de experiencias. En este sentido, es importante hacer una valoración sobre el coste del instrumento, lo que nos llevará a priorizar una de sus finalidades: identificar el mayor número de experiencias posibles o promover la reflexión sobre la EpDCG. Entendemos que nuestra prioridad es la segunda, siendo conscientes de que el mapa elaborado tendrá que ser dinámico y en construcción permanente al tratarse de una realidad viva y cambiante (Corbett y Donehower, 2017). Así, en la fase actual del proyecto la sala viajará por distintos centros educativos de la provincia de A Coruña, a la vez que se creará un mapa digital interactivo que se nutrirá, tanto de las experiencias mapeadas en las distintas salas, como de las experiencias que otros docentes y agentes quieran visibilizar ${ }^{8}$.

\section{Conclusiones}

Teniendo como marco de referencia los enfoques críticos de la EpDCG y fieles a nuestra trayectoria de investigación, la cartografía social, y en concreto la sala cartográfica, se conciben en este estudio como herramientas idóneas para visibilizar, reflexionar sobre la práctica y crear redes de colaboración. Se muestra así la coherencia de usar este método en un estudio de EpDCG, al existir una confluencia en cuanto a sus fines ya que la reflexión crítica, la colaboración, la participación activa y el empoderamiento definen tanto la cartografía social como la EpDCG (Leivas, 2019). El espacio de encuentro creado en las salas se llenó de contenido tras interesantes procesos de reflexión conceptual y negociación de significados por parte del grupo de investigación inicial. Esa reflexión se abrió a la participación de los agentes educativos y sociales clave, quienes valoraron colaborativamente el instrumento, a la vez que se mapeaban experiencias.

Este estudio no solo aporta pruebas sobre la relevancia de la cartografía social como método de investigación en el campo de la EpDCG profundizando en los beneficios de la colaboración entre actores (Cotelo Guerra, et. al, 2019), sino que además ofrece, por un lado, una propuesta completa y extrapolable de caracterización de experiencias de EpDCG y, por otro lado, un primer mapeo colaborativo de proyectos que se llevan a cabo en Galicia.

${ }^{8}$ El mapa se creará con Emapic, iniciativa tecnológica de cartoLAB (Laboratorio de Cartografía del Grupo de Investigación en Visualización Avanzada y Cartografía en Arquitectura, Ingeniería y Urbanismo, UDC) 


\section{Referencias}

AguADO, G. coord. (2011). Educación para el desarrollo y la ciudadanía global (ESDGC). Guía para su integración en centros educativos. InteRed.

ANDREOTI, V. (2014). "Critical literacy: Theories and practices in development education", Policy and Practice: A Dvelopment Education Review, 14, 12-32.

ARES, P. y RISLE, J. (2013). Manual de mapeo colectivo. Recursos cartográficos críticos para procesos territoriales de creación colaborativa. Buenos Aires: Tinta Limón.

Argibay, M. y Celorio, G. (2005). La educación para el desarrollo. Vitoria-Gasteiz: Servicio Central de Publicaciones del Gobierno Vasco.

BoNl, A. (2012). "Educando para la ciudadanía global. Una experiencia de investigación cooperativa entre docentes y profesionales de las ONGD". ESE. Estudios sobre educación, 23, 63-81.

BONI, A. y LóPEZ, E. (2015). Herramientas para planificar y evaluar prácticas para una ciudadanía global. Intermón Oxfam.

Castanheira, A., Barreto, A., Santos, F. y Silva, M. (2016). "Parcerias para a Educação Global: o caso do Projeto Museo Mundial". Sinergias Diálogos Educativos para a Transformação Social, 3 99-109.

CORBET, J. (2009). Buenas prácticas en cartografía participativa. Fondo Internacional de Desarrollo Agrícola.

Cotelo Guerra, Ma. D., Pérez Crego, Ma. C., Iglesias Salvado, J. L. y Pereiro Gonzalez, Ma. del C. (2019). "Retos y ventajas de la colaboración interinstitucional". En DePALMA, R. coord., La educación para el desarrollo y la ciudadanía global. Una experiencia de investigación-acción participativa (pp. 247-264). Barcelona: Graó.

DEPALMA, R. coord. (2019). La educación para el desarrollo y la ciudadanía global. Una experiencia de investigación-acción participativa. Barcelona: Graó.

DELORS, J. (1996). Learning: the treasure within; report to UNESCO of the International Commission on Education for the Twenty-first Century. Paris. UNESCO Publishing. 
FENDLER, R. (2013). "Becoming-Learner: Coordinates for Mapping the Space and Subject of Nomadic Pedagogy", Qualitative Inquiry 19(10) 786-793.

Fernando, D. y GIRALDO, B. (2016). "Cartografía social pedagógica: entre teoría y metodología". Revista Colombiana de Educación, 70, 247-285.

HANNEY, R. (2018). "Problem topology: using cartography to explore problem solving in student-led group projects", International Journal of Research \& Method in Education, 41(4), 411-432.

IgLESIAS, M. y CARRERAS, I. (2013) La colaboración efectiva en las ONG. Alianzas estratégicas y redes. Barcelona: ESADE, Instituto de Innovación Social. Universidad Ramón Llul.

JeNKINS, A. (2015). "Critical Conversations: Dialogue in Global Citizenship Education". Widening Participation and Lifelong Learning, 17(2) 30-47.

LeIVAS, M. y BoNI, A. (2017). "La investigación colectiva para la transformación social hacia la ciudadanía global y el desarrollo sostenible". Revista Española de Desarrollo y Cooperación, 41, 41-53.

LEIVAS, M. (2019). "From the body to the city: participatory action research with social cartography for transformative education and global citizenship". Educational Action Research, 27(1), 40-56.

Ley Orgánica 8/2013, de 9 de diciembre, para la mejora de la calidad educativa.

Martins, A., MAdeira, E. y Gonçalves, T. (2018). "O caminho e o destino: reflexões a partir de um trabalho colaborativo na área da educação para o desenvolvimento". Sinergias-Diálogos Educativos para a Transformação Social, 7,31-47.

Miles, M., Huberman, M. y Saldaña, J. (2014). Qualitative Data Analysis. A Methods Sourcebook. London: Sage.

Montoya Arango, V., García Sánchez, A. y Ospina Mesa, C. (2013). "Andar dibujando y dibujar andando: cartografía social y producción colectiva de conocimientos". Nómadas, 40, 191-205.

Morales lozano, J. A. y Martín Gutiérrez, A. (2013). "La apertura de los centros educativos a su entorno: los centros de educación secundaria". Perspectiva Educacional, Formación De Profesores, 52(1), 68-96. 
Ortega Carpio, Ma. L. coord. (2014). Educación para el Desarrollo en el espacio universitario. Una guía para la colaboración entre ONGD y Universidad. Córdoba: Universidad Loyola Andalucía.

Ortega Carpio, Mª. L., Cordón Pedregosa, Mª R. y Sianes, A. (2013). “Construyendo buenas prácticas de colaboración en el espacio universitario". En ORTEGA CARPIO, $M^{a}$. L., CoRdón PedREgosa, Ma. R. y SiANes, A. (coords.) Educar para la ciudadanía global en el espacio universitario. Buenas prácticas de colaboración entre ONGD y Universidad (pp. 30-52). Sevilla: Universidad Loyola de Andalucía y Fundación ETEA para el Desarrollo y la Cooperación.

Pérez SalGADO, A. coord. (2016). Informe resultado do proceso de elaboración da Estratexia de Educación para o Desenvolvemento da Cooperación Galega. Dirección Xeral de Relacións Exteriores e coa Unión Europea. Vicepresidencia e Consellería de Presidencia, Administracións Públicas e Xustiza. Xunta de Galicia.

Quintano Nieto, J., Ortega Gaite, S., Tejedor Mardomingo, M. y Ruiz Ruiz, E. (2019). "Análisis-diagnóstico de la Educación para el Desarrollo en contextos escolares rurales de un grupo de países europeos". Revista de Fomento Social, 74(3), 265-284

Roura Exyósito, J., Díaz Aguilar, A. l., Ruiz Blanch, A., Sánchez Carretero, A. y Cortés VÁZQUEZ, C. (2018). "Repensando las prácticas académicas: el diseño colaborativo de un proyecto de investigación a partir de la metodología IAP". Revista de Dialectología y Tradiciones Populares, 2, 407-424.

SIIVERMAN, D. (2001). Interpreting Qualitative Data: Methods for Analyzing Talk, Text and Interaction (2nd ed). London: SAGE.

Sírvent, M. T. y RIGAI, L. (2012). Investigación Acción Participativa. Un desafío de nuestros tiempos para la construcción de una sociedad democrática. Proyecto Páramo Andino. 